\title{
Narrowing of the wide nasal dorsum in the 'minimal-hump' nose: A simplified universal approach
}

\author{
Nabil Fanous MD FRCSC, Yannick G Amar MD
}

\begin{abstract}
$\mathrm{N}$ Fanous, YG Amar. Narrowing of the wide nasal dorsum in the 'minimal-hump' nose: A simplified universal approach. Can J Plast Surg 2005;13(3):139-143.
\end{abstract}

The operative procedure frequently used to narrow the nasal pyramid in the context of a regular rhinoplasty is well known to all rhinoplastic surgeons. It consists of hump removal, followed by lateral osteotomies and medialization of the nasal bones. However, narrowing of a wide nasal pyramid, in the absence of an appreciable dorsal hump, as in the case of an ideal dorsal height or a mild hump, presents a different challenge.

The present article describes a simplified approach to the 'nasal plateau resection', a technique that allows the creation of an open nasal roof, without hump removal and without compromising dorsal projection. This permits the medialization of the lateral nasal bones and the subsequent narrowing of the wide pyramid, while preserving the height of the nasal dorsum as viewed in profile.

\section{Diminution de la largeur de l'arête d'un nez minimalement busqué : Approche universelle simplifiée}

\begin{abstract}
L'intervention chirurgicale souvent utilisée pour réduire la largeur de l'arête du nez dans le contexte d'une rhinoplastie standard est bien connue de tous les rhinoplasticiens. Elle consiste à éliminer la bosse, puis à pratiquer des ostéotomies latérales et une médialisation des os du nez. Par contre, diminuer la largeur de l'arête du nez en l'absence de bosse appréciable, comme dans le cas d'une hauteur de nez idéale ou d'une bosse minime, représente un défi considérable.

Le présent article décrit une approche simplifiée à la « résection du plateau nasal », une technique qui permet de créer une voûte nasale ouverte, sans ablation de bosse et sans altération de la projection dorsale. Cette approche permet la médialisation des os latéraux du nez et la diminution subséquente d'une pyramide large, tout en préservant la hauteur de l'arête du nez vue de profil.
\end{abstract}

Key Words: Plateau; Wide nasal dorsum; Wide nose

$\mathrm{R}$ hinoplasty is one of the most commonly performed cosRmetic surgeries. This is reflected by the large number of publications on the topic, focusing on the various facets of the surgery. One aspect of rhinoplasty, related to the wide nasal dorsum, has been addressed in the past, but did not receive the attention it deserves among the majority of rhinoplastic surgeons.

In regular rhinoplasty, the surgical steps performed to narrow a wide nasal pyramid after the removal of a dorsal hump are well known $(1,2)$. First, a hump reduction is carried out via cartilage trimming and bone resection, leading to the removal of the dorsal hump and the creation of an open roof. Then, lateral nasal osteotomies are performed to mobilize the nasal walls medially, to obliterate the open roof and narrow the dorsum.

However, in a small percentage of rhinoplasties, a wide nasal pyramid is not always associated with a moderate or high dorsal hump. This group of patients presents with a wide nasal dorsum and a wide bridge in the frontal view, but with a straight or slightly raised dorsum in the profile view. In such a case, the rhinoplastic surgeon faces the dilemma of not being able to produce an open roof because the minimal lowering of the nasal dorsum may not be deep enough to create one. Without that open roof, the narrowing of the nasal dorsum is not possible, because the existing bone and cartilage between both lateral walls and the septum will not allow the lateral walls to move inwards, even if lateral nasal osteotomies are performed.
In other words, when faced with a nose that is wide in the frontal view, but has a dorsum with a mild or no hump in the profile view, the surgeon still needs to create an open roof to be able to narrow the nose. This open roof may be obtained by over-lowering the dorsum in the same way a hump is removed, which will allow the narrowing of the nasal pyramid by pushing the nasal walls medially. However, this technique solves one problem to create another: the resulting nose will be narrower in the frontal view, but with a recessed dorsum in the lateral view.

Therefore, in the case of a wide nose with no significant hump, a different technique is needed to create an open roof without unnecessary lowering of the dorsal height.

Multiple authors have tackled this problem in the past $(3-11)$. While the basic principles in the majority of these publications are the same, their approaches are somewhat different in terms of surgical details, practicality and clarity.

Our approach, presented here, follows the same principles outlined by others $(3,4,7)$, but we believe it has the advantage of being easy to understand, simple to execute and may be universally applied to most dorsal width variations. This modified approach was first presented in 1984 (12), and was then later briefly described in Plastic and Reconstructive Surgery (6).

The present article describes the anatomical bases and surgical technique of this approach, followed by an evaluation of the advantages, disadvantages and results. 


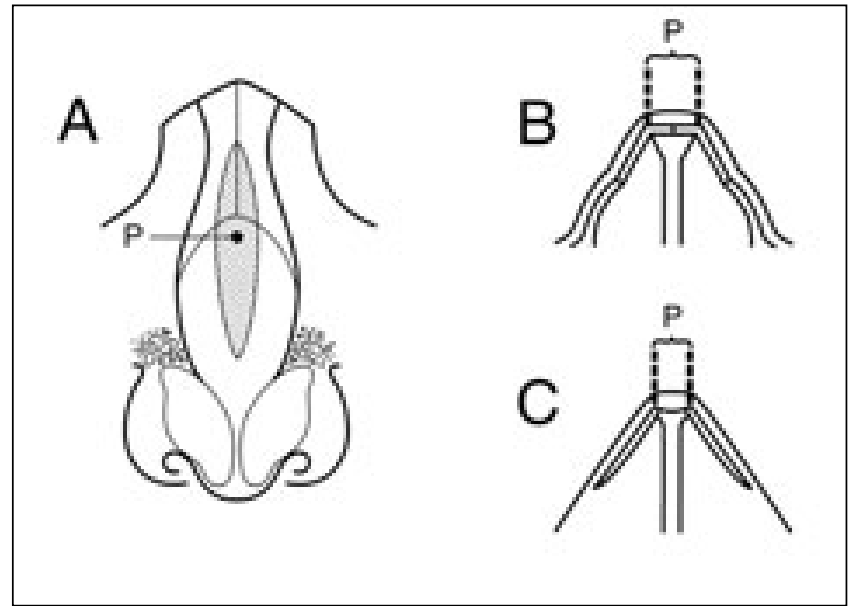

Figure 1) The dorsal plateau. A Frontal view of the plateau $(P)$, demonstrating an elliptical shape (dotted ellipse). It is composed of an upper bony part and a lower cartilagenous part. B Cross section of the bony dorsum, showing the components of the bony plateau. $\mathrm{C}$ Cross section of the cartilagenous dorsum, showing the structure of the cartilagenous plateau
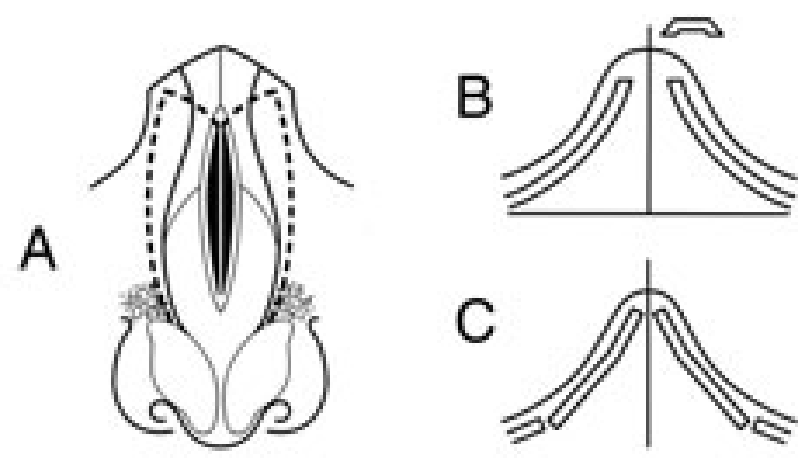

Figure 2) A Removal of a dorsal hump in regular rhinoplasty (plateau excision) leading to an open roof. The dashed lines represent the lateral nasal osteotomies used to infracture the nasal walls and to close the open roof, as well as the optional diagonal osteotomies (short dorsal lines medially). B Cross section of the lateral nasal walls in relation to the midline septum after hump removal, showing an empty space (open roof) on each side of the septum. C Cross section of the infractured nasal walls. The open roof is now closed and the nasal pyramid is narrower

\section{SURGICAL ANATOMY OF THE NASAL DORSUM}

Before describing the surgical technique, some essential anatomical concepts must be reviewed $(6,13,14)$.

On careful inspection, one notices that the nasal septum is not a straight plate with two parallel surfaces (Figure 1C). Instead, the septum progressively widens as it approaches its dorsal border. Thus, in a vertical cross section, the septum looks like a palm tree, starting at the vomer junction as a thin plate of cartilage with parallel walls, then widening near the dorsum to form a flat surface (or plateau). This cartilagenous plateau extends along the entire length of the dorsal border of the cartilagenous septum (Figure 1A, dotted ellipse 'P').

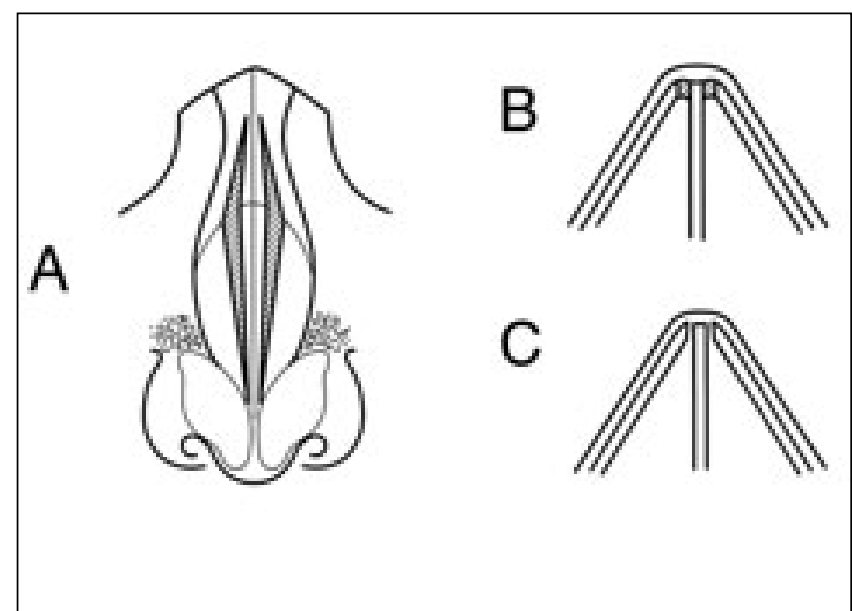

Figure 3) A Plateau resection. The paramedian portions of the nasal plateau (striated wedges of cartilage and bone) are removed, while the midline portion (nasal septum) is left intact. B Cross section showing the paramedian parts of the plateau to be resected. C Lateral nasal walls after inward mobilization

In the superior half of the nasal dorsum, the bony plateau (Figure 1B, surface P) is formed by the extension of both lateral bony walls medially over the bony septum. In other words, as the nasal bones approach the midline, they curve medially, forming a flat surface along the nasal dorsum, a sort of bony platform, or 'bony plateau', which is continuous with the previously described cartilagenous plateau.

This complete nasal plateau (Figure 1A, dotted ellipse 'P') is therefore composed of an upper bony part and a lower cartilagenous part. When viewed from a frontal view, this plateau is elliptical in shape, and extends from the root of the nose to the supratip area (septal angle). Its widest portion is located at the rhinion, a point where its lower cartilagenous half meets its upper bony one (Figure 1A). This plateau is not really flat, but rather depressed along its central line in the form of a midline groove. The width of the nasal plateau determines the width of the nasal pyramid at the dorsum.

\section{SURGICAL TECHNIQUE}

The concept of plateau resection

The usual method of narrowing a wide nasal pyramid in regular rhinoplasty starts by creating an open roof secondary to dorsal hump removal (Figure 2A, B). This open roof provides the space necessary for the inward movement of the lateral nasal walls following lateral nasal osteotomies (Figure 2C). However, in the absence of a hump or in the presence of a mild one, any attempt to create an open roof by over-resecting the nasal dorsum will result in a lower than ideal nasal profile or a saddle deformity. Therefore, a different technique must be found to create the 'open roof' necessary for the narrowing of the wide dorsum, but without unnecessary alteration of the height of that dorsum.

This technique, termed plateau resection, consists of removing both paramedian portions (right and left) of the nasal plateau, while leaving the middle portion intact (Figure 3A, B). This results in the opening of the roof of the nose without altering the height of the septum, thus preserving dorsal projection. In fact, what is being removed is the flared portion of the nasal septum (in the cartilagenous portion, Figure 1C), as 
well as the flat surfaces created by the nasal bones (in the bony portion, Figure 1B), while leaving a thinner and intact septum in the midline, with two empty spaces on the left and right (Figure 3A, shaded triangles). In other words, this procedure is a partial plateau removal or 'plateau resection', rather than a complete plateau removal or 'plateau excision' (as in the nasal hump removal).

\section{Preoperative marking}

The two paramedian portions (left and right) of the nasal plateau that have to be removed are marked on the dorsal skin (Figure 3A, shaded triangles). The lateral outline of each paramedian portion coincides with the lateral border of the plateau.

\section{The technique}

There are simply two basic steps to the nasal plateau resection: first, medial and lateral cartilagenous incisions are performed in the lower cartilagenous part of the plateau, which is then followed by medial and lateral bony osteotomies in the upper bony part of the plateau (Figure 3A).

To begin with, exposure of the nasal dorsum through skin undermining may be achieved by an open $(8,14-16)$ or closed approach (6) (we use the latter). If the dorsum shows a mild hump, it is rasped down or resected to the ideal dorsal height. At this point, a closed mosquito is introduced inside each nasal cavity to check for the presence of an open roof. If the free hand on the outside of the nose can feel the mosquito under the dorsal skin, the roof is opened. If this is not the case, the plateau resection technique is then started.

The first step in resecting the plateau is to perform two cartilagenous incisions in its inferior portion. A number 15 scalpel blade, with its blade facing up (dorsally), is placed high in the ceiling of the right nasal cavity, on the deep surface of the dorsum and flush with the nasal septum. The scalpel is then used to perform the medial cartilagenous incision on that side, through the nasal mucosa and dorsal septal cartilage, starting at the rhinion (the uppermost point of the cartilagenous plateau) and moving downwards toward the septal angle (the lowermost point of the cartilagenous plateau). The surgeon moves the blade carefully in a sawing-like fashion (back and forth), cutting through mucosa and cartilage along the medial border of the cartilagenous wedge that has to be excised on the right side (Figure 3A, striated lower triangle). Throughout this procedure, the surgeon's free hand is placed over the dorsum to gently palpate the skin and confirm the position of the blade. The lateral cartilagenous incision is then performed on the same right side, with the scalpel again facing dorsally. This lateral incision also extends from the osteocartilagenous junction in a sawing-like fashion toward the septal angle. The cutting blade moves along the lateral border of the same cartilagenous wedge, at the junction of the flaring cartilagenous septum and the upper lateral cartilage.

In the second step, the osteotomies of the upper bony half of the right plateau are addressed. A straight flat osteotome, $2 \mathrm{~mm}$ to $5 \mathrm{~mm}$ in width, is placed high inside the right nasal cavity, flush against the nasal septum and at the level of the rhinion. The osteotome is then used to perform a medial plateau osteotomy extending from the osteocartilagenous junction (the lowest point of the bony plateau) to the root of the nose (the highest point of the bony plateau). As the flat osteotome advances upward, it cuts through mucosa and bone
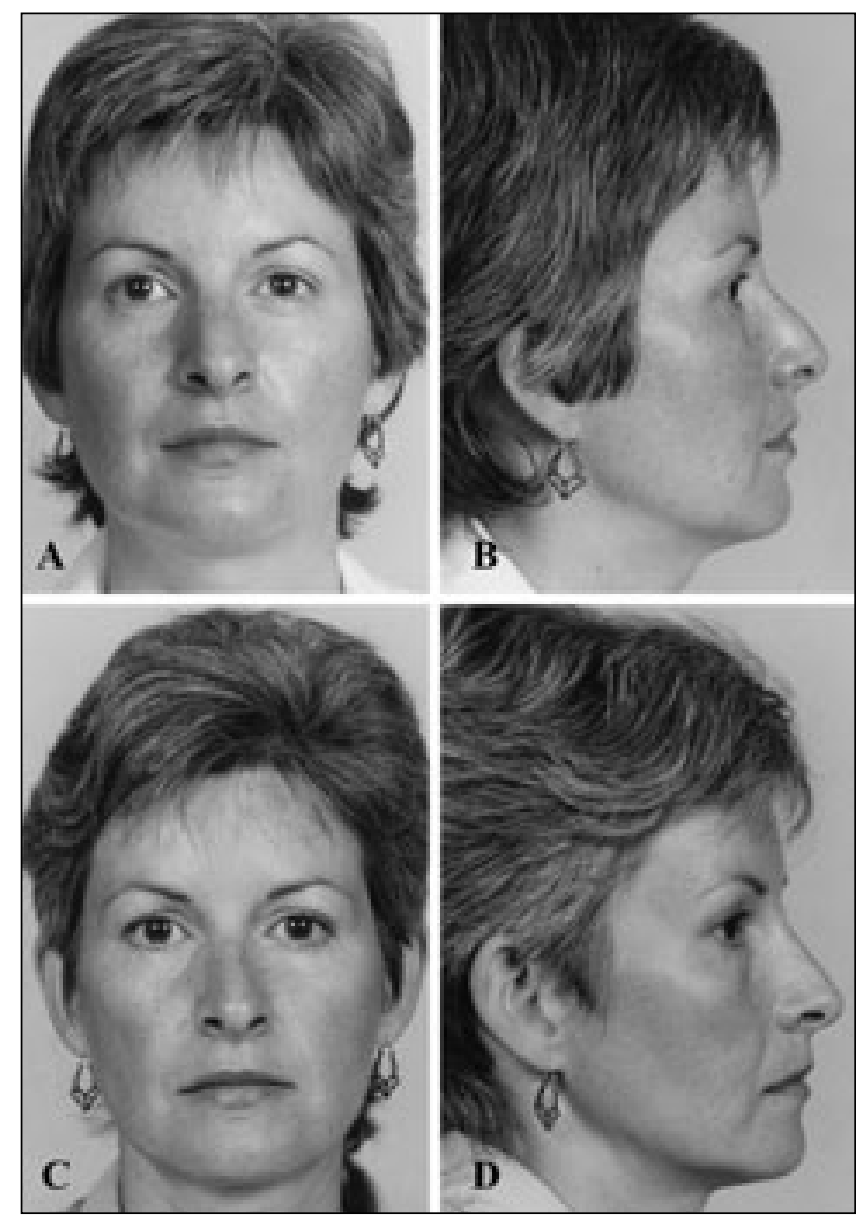

Figure 4) A and B Preoperative photographs of a 38-year-old woman who underwent a previous rhinoplasty elsewhere and presented with a main complaint of a persistent wide nasal plateau, a mild dorsal hump and a tip recession that gave the impression of a pseudohump. $\mathbf{C}$ and $\mathbf{D}$ Postoperative photographs 12 months after revision rhinoplasty using minimal dorsal rasping, bilateral plateau resection and tip augmentation with a dacron fabric implant

along the medial border of the right bony wedge that has to be excised (Figure 3A, striated upper triangle). The surgeon must be careful to keep the osteotome completely flat against the septum throughout the osteotomy, because if the osteotome is not parallel to the bony septum, it may advance into the septum, inadvertently cutting it and lowering its height. A lateral plateau osteotomy is then performed at the lateral border of the same bony wedge, using the same technique. As in the case of the cartilagenous incisions, the surgeon's free hand is used externally for securing the position of the osteotomy lines. The combined cartilagenous incisions and bony osteotomies result in the delineation of a complete right triangular wedge (which is actually formed of two attached wedges of cartilage and bone).

The above two major steps (cartilagenous incisions and bony osteotomies) are then repeated on the left side of the nasal plateau. The two loose triangular bony and cartilagenous wedges (left and right) are then pulled out of the nose using a straight hemostat. Any resistance to this pulling manoeuvre indicates the presence of an incomplete cartilagenous incision or an incomplete bony osteotomy. This will necessitate going 


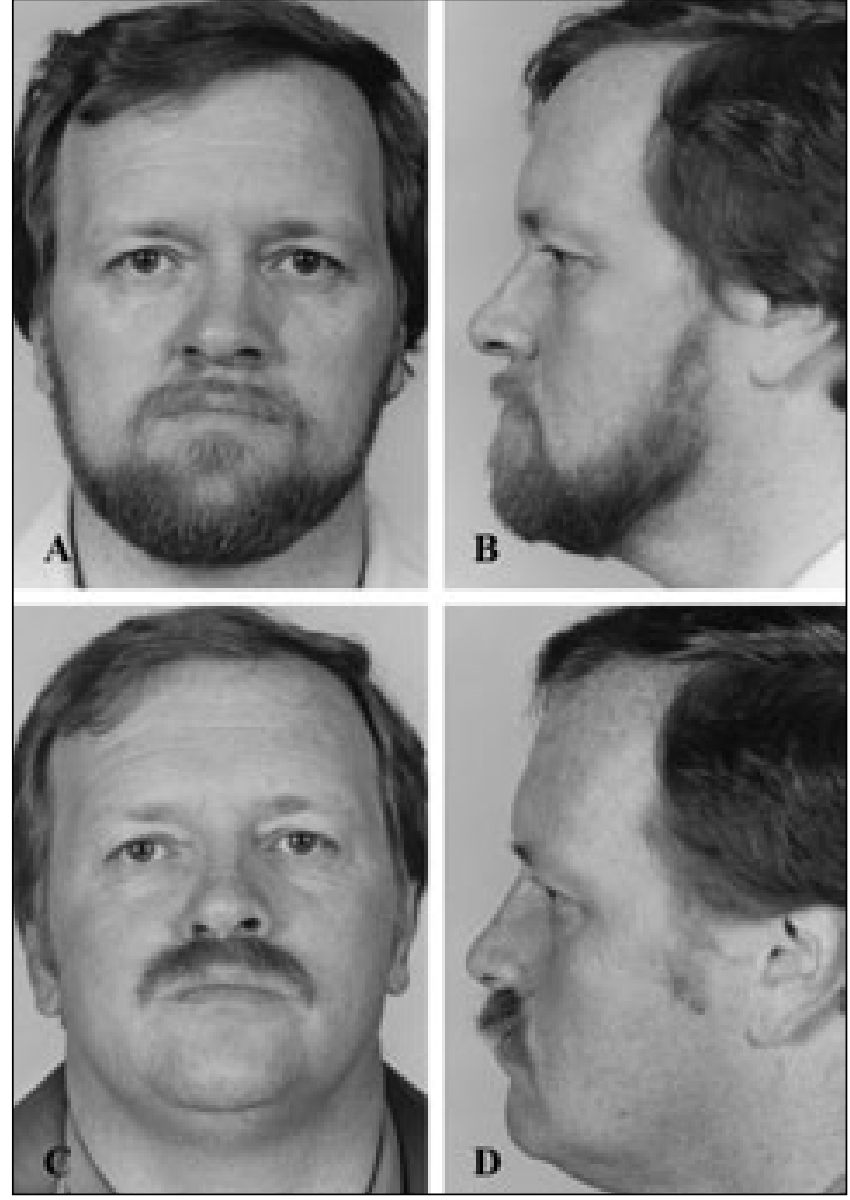

Figure 5) A and B Preoperative photographs of a 40-year-old man with a localized unilateral widening of the left nasal plateau, as well as a slightly recessed (low) nasal dorsum. C and D Postoperative photographs 16 months following rhinoplasty with left plateau resection, as well as dorsal augmentation with a cartilagenous graft
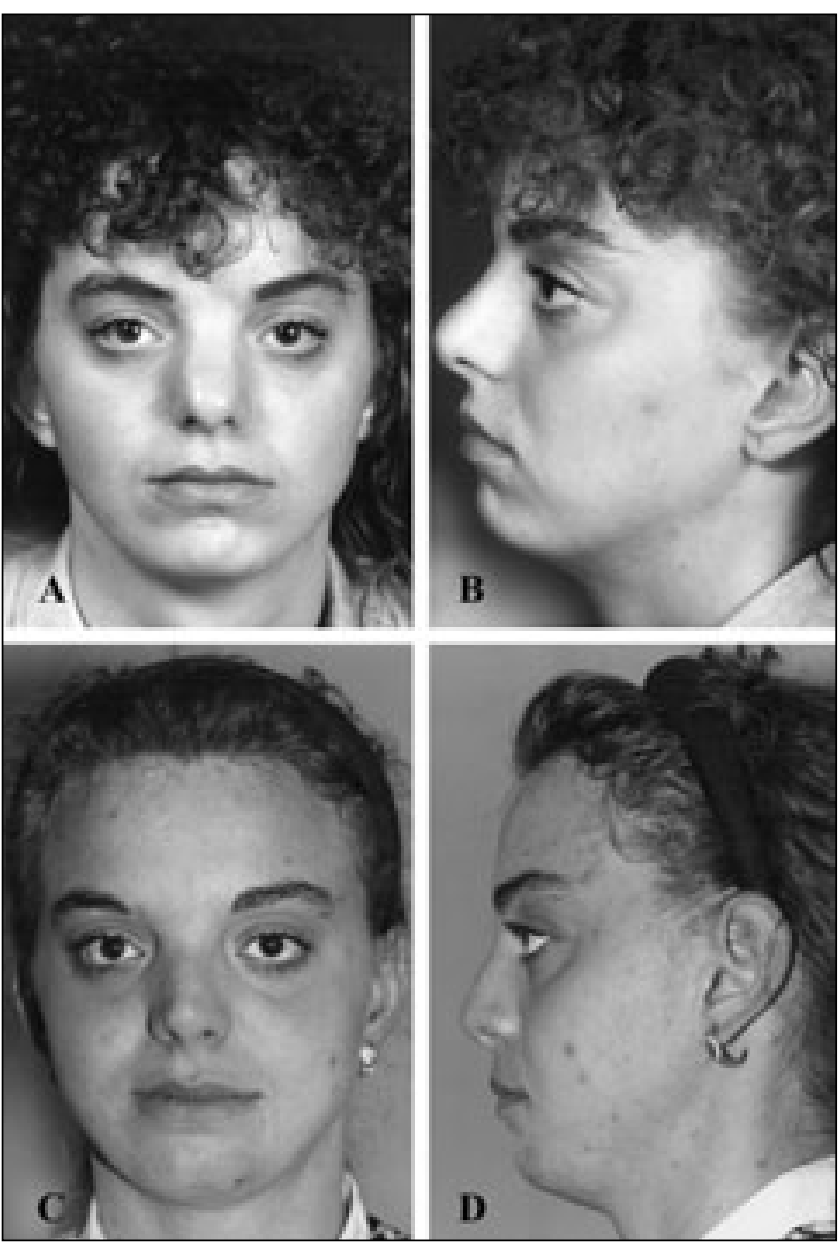

Figure 6) A and B Preoperative photographs of a 20-year-old woman with an extremely wide nasal plateau and a mild cartilagenous hump. $\mathbf{C}$ and $\mathbf{D}$ Postoperative results 29 months after a rhinoplasty with bilateral plateau resection. The surgeon felt this result could be further enhanced with a minimal tip augmentation in a secondary tip revision, but the satisfied patient declined the offer

(median duration of 28 months). Thirteen patients underwent primary rhinoplasty, while three patients were previously operated on elsewhere and came for a secondary procedure. These revision cases were not particularly different from the primary ones with regard to the plateau resection complexity. All plateau resections were bilateral, except for four cases. While the majority of patients had a mild hump deformity, they still needed plateau resection because removal of the minimal hump was not sufficient to produce an open roof. Three patients had a small dorsal augmentation, in addition to the plateau resection, to correct a preoperative slightly low dorsum and to enhance the illusion of a narrower nasal pyramid. Figures 4, 5 and 6 show preoperative photographs and postoperative results of the plateau resection technique. Figure 4 is a common example of the plateau resection technique, even though it is a revision case. Figure 5 is an example of the unilateral plateau resection, which was also combined with a dorsal graft. Figure 6 is an example of a plateau resection on an unusually wide dorsum. 


\section{DISCUSSION}

The standard procedure of dorsal hump removal combined with lateral nasal osteotomies to narrow the wide nasal pyramid in the presence of a dorsal hump is adequate in the great majority of rhinoplasties. However, in the rare context of a wide nasal dorsum with little or no hump, this procedure may not be applicable. Dorsal height, in the profile view, is mostly determined by the height of the nasal septum. In the case of a cosmetically satisfying or minimally projecting dorsal profile before surgery, nasal height must be preserved. If such a case is associated with a wide nasal dorsum, it will be necessary to create an open nasal roof to allow the medial movement of the lateral nasal walls. The nasal plateau resection described in the present article creates an open roof on both sides of the nasal septum, allowing inward movement of the nasal walls, while preserving septal height at the same time.

This idea is not new and has been covered by a number of authors in the past. Most of these publications have followed the same basic concepts, but their approaches were different in terms of surgical steps, algorithms, classifications, etc.

We believe the above-described approach to the plateau resection, in the occasional wide nose with no or minimal hump, can be a helpful tool in the hands of the average rhinoplastic surgeon.

ACKNOWLEDGEMENTS: The authors express their thanks to Ildico Horvath, medical artist, at Montreal General Hospital, for her assistance in preparing the artwork; Robert Derval, medical photographer, for his photographic contribution; Barbara Armbruster MA, for the editing; Amanda Fanous for the literature search; Stephanie Luetticken MBA, for organizing the manuscript; and Joelle Bejjani for the typing.

\section{REFERENCES}

1. Ishida J, Ishida LC, Ishida LH, Vieira JC, Ferreira MC. Treatment of the nasal hump with preservation of the cartilaginous framework. Plast Reconstr Surg 1999;103:1729-33; 1734-5.

2. Fenton RS. Surgery of the nasal dorsum. J Otolaryngol 1978;7:24-8.

3. Sheen JH, Sheen AP. Aesthetic Rhinoplasty. St Louis: Mosby-Year Book Inc, 1987.

4. Daniel RK. Aesthetic Plastic Surgery: Rhinoplasty. Boston: Little Brown \& Co, 1993.

5. Prendiville S, Zimbler MS, Kokoska MS, Thomas JR. Middle-vault narrowing in the wide nasal dorsum: The "Reverse Spreader" technique. Arch Facial Plast Surg 2002;4:52-5.

6. Fanous N. Unilateral osteotomies for external bony deviation of the nose. Plast Reconstr Surg 1997;100:115-23.

7. Harris MO, Baker SR. Management of the wide nasal dorsum. Arch Facial Plast Surg 2004;6:41-8.

8. Kabaker SS. An approach to aesthetic rhinoplasty in the noncaucasian nose. Arch Otolaryngol 1977;103:461-7.

9. Man A. Surgical reduction of the broad nose. Am J Otolaryngol 1980;1:446-50

10. Hubbard TJ. Bridge narrowing in ethnic noses. Ann Plast Surg 1998;40:214-8.

11. Jeppesen F. Anterior wedge excision in correcting deflections of the nasal dorsum. Ear Nose Throat J 1992;71:49-52; 55-8.

12. Fanous N. Unilateral osteotomies for external bony deviation of the nose. Presented at the annual meeting of the Canadian Society of Otolaryngology - Head \& Neck Surgery, Halifax, Canada, June 1984.

13. Tardy ME. Surgical Anatomy of the Nose. New York: Raven Press, 1990.

14. Grymer LF, Gregers-Petersen C, Baymler Pedersen H. Influence of lateral osteotomies in the dimensions of the nasal cavity. Laryngoscope 1999;109:936-8.

15. Goodman WS, Charbonneau PA. External approach to rhinoplasty. Laryngoscope 1974;84:2195-201.

16. Mayer TG, Fleming RW. The nasal dorsum - open approach. Facial Plast Surg 1988;5:143-59. 